\title{
pharma-kritik
}

AZB 9500 Wil

Jahrgang 31

Nummer 20/2009

Lohnt es sich? (E. Gysling) .... 77

Hinweis: Neue Website ..... 78

Sachverzeichnis zum Jahrgang 31 79

\section{ceterum censeo}

\section{Lohnt es sich?}

Wie noch nie stehen uns heute medizinische Informationen in fast unerschöpflicher Menge zur Verfügung. Vieles ist kostenlos über das Internet abrufbar. Auch Informationen zu neuen Medikamenten sind reichlich vorhanden. So ergibt Ende Juli 2010 eine Suche nach «Saxagliptin» in der medizinischen Datenbank Medline zwar lediglich 63 Treffer, eine Google-Suche mit demselben Begriff spricht jedoch von «ungefähr 58‘700» Ergebnissen. Sucht man mit dem Markennamen «Onglyza», so findet man gar über 75`000 Ergebnisse. Dies mag auf den ersten Blick faszinieren; sieht man die Google-Resultate genauer an, entwickelt sich aber schnell eine Art von Daten-Albtraum. Neben Resultaten, die wahrscheinlich objektiv korrekt sind, aber kaum mehr aussagen als beispielsweise die im Arzneimittel-Kompendium enthaltenen Basisinformationen, finden sich fast beliebig viele Pseudo-Resultate, die überhaupt nichts Brauchbares zu Saxagliptin vermitteln oder auch offensichtlich Falsches kolportieren. Grundsätzlich ist es jedoch durchaus möglich, die Spreu vom Weizen zu trennen, d.h. durch Eingabe zusätzlicher Suchbegriffe und mittels sorgfältigem Studium der relevant erscheinenden Resultate brauchbare Aussagen zu Saxagliptin «herauszufischen».

Somit stellt sich die Frage: Lohnt es sich denn, für ein mageres Blatt wie die «pharma-kritik» jährlich rund 100 Franken zu bezahlen? Aus meiner Sicht gibt es zwei wesentliche Argumente, weshalb dieses Geld sinnvoll eingesetzt ist.

Das erste Argument heisst: Zeit. Ein «pharma-kritik»-Text ist nicht nur kurz, sondern setzt zudem ein Hauptaugenmerk auf Aspekte, die in offiziellen Texten leicht zu kurz kommen oder im Wust der mehr oder weniger bedeutsamen Informationen untergehen. Mit anderen Worten: der Text vermittelt innerhalb von kurzer Zeit Wissen, das man sonst mit stundenlangem Aufwand zusammentragen müsste. Tatsächlich benötigen wir in unserem Redaktionsteam in der Regel nicht nur Stunden, sondern - je nach Thema - Tage bis Wochen, bis wir nur schon das Wichtigste gefunden und geordnet haben. (Damit ist ein Text allerdings noch nicht geschrieben.)

Das zweite Argument heisst: Kompetenz. Zu Medikamenten und generell zu therapeutischen Fragen gibt es immer verschiedene Aussagen und Fakten, die gewertet werden müssen. Dabei genügt es nicht, Internet-Suchmaschinen und medizinische Datenbanken abzufragen. Um sich ein möglichst vollständiges Bild $\mathrm{zu}$ einem Thema $\mathrm{zu}$ machen, ist meistens die Lektüre von spezialisierten Zeitschriften notwendig. Ebenfalls nützlich, wenn auch zeitraubend, kann das Studium von offengelegten Dokumenten und Protokollen von Arzneimittelbehörden (insbesondere von Texten der amerikanischen Behörde FDA) sein. Von Bedeutung ist ferner der Informationsaustausch innerhalb der internationalen Gruppierung von unabhängigen ArzneimittelZeitschriften («International Society of Drug Bulletins»). Schliesslich verschafft erst ein Redaktionsteam, das sich nicht nur an pharmakologisch-pharmazeutischen Aspekten orientiert, sondern auch mit dem Alltag der medizinischen Praxis vertraut ist, den Texten Relevanz und Vertrauenswürdigkeit.

Dass wir in dieser Zeitschrift nicht Kritik um der Kritik willen veröffentlichen, sondern nur im Interesse der Sache bzw. des kranken Menschen, dürfte allen Leserinnen und Lesern wohl bewusst sein.

Stellt man die Frage «Lohnt es sich?» aus der Sicht des Redaktionsteams und des Verlags, so ist die Antwort nicht ganz so einfach. Der primäre Markt unserer Zeitschriften pharma-kritik und infomed-screen - sind ja die Angehörigen der Gesundheitsberufe in der Deutschschweiz. Dazu kommt natürlich noch eine kleine Zahl von Abonnentinnen und Abonnenten in der französischen Schweiz, im Tessin und im deutschsprachigen Ausland. Im Vergleich mit anderen werbefreien Arzneimittel-Publikationen in Europa erreichen wir jedoch auch bei «grosser Beliebtheit» nur eine lächerlich kleine Auflage. Es ist uns selbstverständlich bewusst und wir freuen uns auch darüber, dass uns unsere Leserinnen und Leser ungewöhnlich viel Wohlwollen entgegenbringen. Dieses Wohlwollen spüren wir auch, wenn wir für die Stiftung info-pharma freiwillige Beiträge - besonders grosszügig vom Aroser Kongressverein - erhalten. 
(Dank der Stiftung info-pharma konnte z.B. jetzt unsere Website, auf der wir einen grossen Teil unserer Publikationen kostenlos zur Verfügung stellen, neu gestaltet werden.) Der Publikations-Alltag kann und soll aber nicht aus dem relativ bescheidenen Stiftungsgeld finanziert werden.

Die Anzahl Abonnemente, die wir verkaufen können, nimmt jedoch schon seit ein paar Jahren ab. Besonders weil es je länger desto weniger Einzelpraxen gibt, dient ein Abonnement oft mehreren Kolleginnen und Kollegen. (Der höhere Preis der Abos für Gruppenpraxen kompensiert dies nur in geringem Ausmass.) So müssen wir zur Zeit damit leben, dass unser Markt kontinuierlich kleiner und damit «unrentabler» wird. Ob und wie sich dies in Zukunft ändern lässt, darüber diskutieren wir natürlich im Team - einfache Patentlösungen gibt es kaum.

Etzel Gysling

\section{Hinweis}

\section{Neue Infomed-Website}

Seit Ende Juli 2010 ist die neue Version unseres InternetAngebotes (www.infomed.org) aufgeschaltet. Wir möchten Sie einladen, sich selbst ein Bild von den Vorzügen der neuen Gestaltung zu machen und die Website auch rege zu benutzen. Abonnentinnen und Abonnenten haben wie bisher Zugang zu den Volltexten unserer Zeitschrift. Neu sind noch mehr frühere Jahrgänge (bis zum Jahr 1988) abrufbar; auch können die einzelnen Nummern jetzt in Form von PDFDateien auf den Computer geholt werden.

Schon bisher konnten auf unserer Website aktuelle Meldungen zu Arzneimittelproblemen unter dem Titel «Bad Drug News»» konsultiert werden - das entsprechende Archiv reicht bis ins Jahr 2000 zurück. Dieses Archiv illustriert in kurzen Texten die zahlreichen «Arzneimittel-Unglücksfälle», die sich im vergangenen Jahrzehnt ereignet haben. Obwohl wir zur Zeit nicht zu viel versprechen möchten, besteht die Absicht, in Zukunft die «Bad Drug News» durch weitere aktuelle Meldungen zu ergänzen. Schliesslich müssen nicht nur die «schlechten», sondern auch die «guten» Nachrichten berücksichtigt werden. Wie bis anhin sollen aktuelle Kurzmeldungen jedermann zugänglich sein.

Über die (ebenfalls verbesserte) Suchfunktion der Website lassen sich ältere und neuere Texte finden. Die neueren Dokumente sind allerdings nur zugänglich, wenn man über ein Abo-Passwort verfügt. Bitte beachten Sie, dass die bisherigen Passwörter auf der neuen Website nicht mehr gültig sind. Ihr Benutzername entspricht wie bisher Ihrer E-MailAdresse. Wer sich noch nicht auf der neuen Website eingetragen hat, möge bitte den (neuen) Passwort-Antrag ausfüllen (www.infomed.org/pw request.php). Um Zugang zu erhalten, benötigen Sie Ihre Abonummer, die Sie auf der Versandhülle oder auf der Rechnung finden. Diejenigen, die sowohl pharma-kritik als auch infomed-screen abonniert haben, sollten sich möglichst gleichzeitig für beide Zeitschriften anmelden - dabei sind beide Abonummern einzutragen. Nachher genügt ein Passwort, um beide Zeitschriften im Volltext online konsultieren zu können. In beiden Zeitschriften stehen viele Hunderte von Texten in deutscher Sprache zur Verfügung: pharma-kritik informiert kritisch über alles, was beim Verschreiben wichtig ist und infomedscreen bietet kommentierte Zusammenfassungen wichtiger klinischer Studien. Da alle Texte datiert sind, lässt sich der Aktualitätsgrad zuverlässig abschätzen. Die Suchresultate können nach der Relevanz oder nach dem Erscheinungsdatum geordnet werden. Viele ältere Texte erweisen sich noch als sehr brauchbar.

Ein vergessenes Passwort erfordert übrigens jetzt keinen grossen Aufwand mehr: mit Hilfe des Benutzernamens (EMail-Adresse) kann jederzeit ein neues Passwort angefordert werden. Dieses sollte sich in der Regel schon nach wenigen Minuten in der Mailbox finden.

Sollten Sie auf unserer Website Fehler oder Probleme entdecken, so sind wir Ihnen für eine kurze Mitteilung (via Kontaktformular im Internet oder über 071-910-0866 telefonisch) dankbar. Wir sind aber auch gerne bereit, Ihnen auf demselben Weg zu helfen, wenn Sie nicht korrekt Zugang erhalten oder bei der Verwendung der Website auf andere Schwierigkeiten stossen.

Für Personen, die unsere Zeitschriften nicht abonniert haben, stellen wir seit dem Wechsel auf die neue Website von beiden Zeitschriften noch mehr (ältere) Jahrgänge gratis zur Verfügung. Diese Dienstleistung ist mindestens teilweise der finanziellen Unterstützung durch die Stiftung infopharma zu verdanken. Viele Abonnentinnen und Abonnenten haben schon Beiträge an diese Stiftung geleistet und damit geholfen, dass unabhängige medizinische Information möglichst weit verbreitet werden kann. Wir sind stolz darauf, dass sich so viele mit uns zusammen für eine gute und sinnvolle Aufgabe engagieren.

Selbstverständlich steht die Auskunftsfunktion, die mit der Stiftung verbunden ist, weiterhin zur Verfügung. Sie erreichen uns nach wie vor über das Kontaktformular im Internet, telefonisch, per Fax oder auch per Post (VerlagsAdresse).

Infomed-Verlag

\section{Dank}

Mit dieser Nummer ist der Jahrgang 31 (2009) abgeschlossen. Ich danke allen Abonnentinnen und Abonnenten, den Mitarbeiterinnen und Mitarbeitern und den vielen Fachleuten, die pharma-kritik-Texte verfasst oder durchgesehen haben, ganz herzlich.

Etzel Gysling 